This document was prepared as an account of work sponsored by the United States Government. While this document is believed to contain correct information, neither the United States Government nor any agency thereof, nor The Regents of the University of California, nor any of their employees, makes any warranty, express or implied, or assumes any legal responsibility for the accuracy, completeness, or usefulness of any information, apparatus, product, or process disclosed, or represents that its use would not infringe privately owned rights. Reference herein to any specific commercial product, process, or service by its trade name, trademark, manufacturer, or otherwise, does not necessarily constitute or imply its endorsement, recommendation, or favoring by the United States Government or any agency thereof, or The Regents of the University of California. The views and opinions of authors expressed herein do not necessarily state or reflect those of the United States Government or any agency thereof or The Regents of the University of California. 


\title{
HIGH ENERGY DENSITY PHYSICS EXPERIMENTS WITH INTENSE HEAVY ION BEAMS*
}

F.M. Bieniosek ${ }^{\#}$, E. Henestroza, M. Leitner, B.G. Logan, R.M. More, P.K. Roy, P. Ni, P.A. Seidl, W.L. Waldron, Lawrence Berkeley National Laboratory, Berkeley CA; J.J. Barnard, Lawrence Livermore National Laboratory, Livermore CA and HIFS-VNL, U.SA.

\begin{abstract}
The US heavy ion fusion science program has developed techniques for heating ion-beam-driven warm dense matter (WDM) targets. The WDM conditions are to be achieved by combined longitudinal and transverse space-charge neutralized drift compression of the ion beam to provide a hot spot on the target with a beam spot size of about $1 \mathrm{~mm}$, and pulse length about 1-2 ns. As a technique for heating volumetric samples of matter to high energy density, intense beams of heavy ions are capable of delivering precise and uniform beam energy deposition $\mathrm{dE} / \mathrm{dx}$, in a relatively large sample size, and the ability to heat any solid-phase target material. Initial experiments use a $0.3 \mathrm{MeV} \mathrm{K}+$ beam (below the Bragg peak) from the NDCX-I accelerator. Future plans include target experiments using the NDCX-II accelerator, which is designed to heat targets at the Bragg peak using a 3-6 MeV lithium ion beam. The range of the beams in solid matter targets is about 1 micron, which can be lengthened by using porous targets at reduced density.

We have completed the fabrication of a new experimental target chamber facility for WDM experiments, and implemented initial target diagnostics to be used for the first target experiments in NDCX-1. The target chamber has been installed on the NDCX-I beamline. The target diagnostics include a fast multi-channel optical pyrometer, optical streak camera, VISAR, and high-speed gated cameras. Initial WDM experiments will heat targets by compressed NDCX-I beams and will explore measurement of temperature and other target parameters. Experiments are planned in areas such as dense electronegative targets, porous target homogenization and two-phase equation of state.
\end{abstract}




\section{INTRODUCTION}

The US heavy ion fusion science program is developing techniques for heating ion-beamdriven warm dense matter (WDM) targets [1-4]. Intense ion beams have several attractive features as a technique for generating WDM. These features include:

-Precise control of local beam energy deposition $\mathrm{dE} / \mathrm{dx}$, nearly uniform throughout a given volume, and not strongly affected by target temperature,

-Large sample sizes (about 1 micron thick by $1 \mathrm{~mm}$ diameter),

-The ability to heat any target material, for example, foams, powders, conductors, insulators, solid, gas, etc.

Uniformity of target heating and efficiency of beam energy deposition are obtained by heating with the Bragg peak at the center of the target. This approach allows operation with moderate beam energy $(\sim 1 \mathrm{MeV} / \mathrm{AMU})$. Other scenarios take advantage of two other regions where the $\mathrm{dE} / \mathrm{dx}$ curve for heavy ions is nearly flat: high energy approaching $1 \mathrm{GeV} / \mathrm{AMU}$ (as at GSI [5]), and low energy $\sim 10 \mathrm{keV} / \mathrm{AMU}$. The range of the low and moderate energy beams planned for the HIFS-VNL experiments is about 1 micron in solid matter targets, which can be lengthened by using porous targets at reduced density. Because of the short range, it is necessary to compress beam pulses to roughly $1 \mathrm{~ns}$ to be consistent with the hydrodynamic expansion time of the target. The range can be extended by heating low-density porous targets, for example density in the range of $1-10 \%$ of solid density, extends ion beam range and hydrodynamic expansion time by factors of 10-100. Initial experiments will be at low beam velocity, below the Bragg peak using the existing NDCX-1 accelerator $(0.3-0.4 \mathrm{MeV} \mathrm{K}+)$. Intense ion beam currents must be focused to a spot size less than $1 \mathrm{~mm}$ in order to achieve sufficient heating power on target for these low kinetic energy ions. This is only made possible by the neutralized drift compression technique recently developed in the HIFS-VNL. The ion beam will undergo combined longitudinal and transverse neutralized drift compression to provide a hot spot on the target with a beam spot size $\leq 1 \mathrm{~mm}$, and pulse length about 1-2 ns [4].

Figure 1 illustrates schematically the required components for such warm dense matter heating experiments.

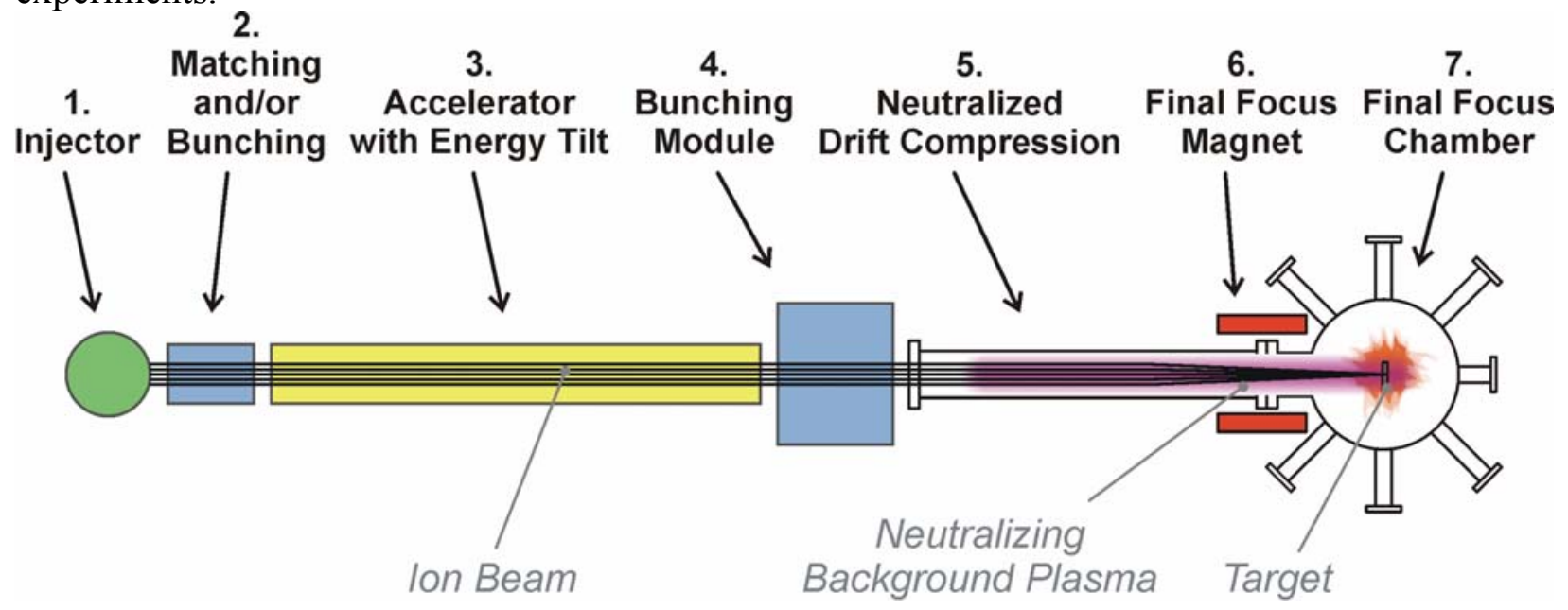

Figure 1: Experimental setup for heavy-ion heated WDM targets. 
This approach has significant consequences for the experimental target and target chamber setup. The target chamber as built is designed to provide:

1. sufficiently dense $\left(10^{12}\right.$ to $\left.10^{14} \mathrm{~cm}^{-2}\right)$ plasma injection to neutralize the space charge of the incoming ion beam,

2. a strong (8 Tesla) final focus solenoid for radial ion beam compression,

3. vacuum pumping,

4. retractable ion beam diagnostics,

5. a retractable target holder with in-situ alignment capabilities,

6. fast optical target diagnostics with access to the front, side and back of the target foil,

7. accurate (micron range) alignment capabilities

We have developed a WDM target chamber and a suite of target diagnostics including a high speed multi-channel optical pyrometer, optical streak camera, VISAR, and high-speed gated cameras [6-8]. The target chamber and diagnostics will be installed downstream of the induction bunching module on NDCX-1 (Fig. 2).

Initial WDM targets will provide the opportunity to gain experience with diagnostics for WDM targets. The targets will be approximately a range thick, for example 350-nm Al and 150-nm Au. Simulations of target heating using HYDRA indicate heating to approximately $0.2 \mathrm{eV}$, depending on beam final focus parameters achieved in initial experiments. We have begun a series of planned experiments in warm dense matter. These experiments include a 2006 porous target experiment at GSI to compare response of solid and porous targets, and planned or possible experiments at NDCX-1 or NDCX-2 such as

-initial beam-driven target experiments

-low density porous targets

-high electron affinity (e.g. halogen) target study

-two-phase liquid/vapor targets to study fragmentation and droplet formation

-beam - shock wave coupling in cryogenic targets

-EOS studies

The following sections describe in detail the subcomponents of the target chamber facility and diagnostics.

\section{TARGET CHAMBER}

Figure 2 displays a schematic view of the target chamber facility with the major components highlighted. The longitudinally compressing ion beam enters from the left and is focused to less than $1 \mathrm{~mm}$ diameter by a strong, pulsed solenoid. Because of the low energy of the incoming ion beam the focal length of the final focus solenoid is short $(\sim 40 \mathrm{~cm})$. This puts a major constraint on the target chamber design since each sub-component has to be tightly packed in space. 
The target chamber is a precisely machined octagon made of 304 stainless steel as shown in Fig. 2. The octagonal shape allows the precise arrangement of different diagnostics around the side of the target which is located in the exact center of the chamber. In addition, the front entrance flange as well as the exit flange provide 4 vacuum ports each which allow to look straight through the target plane.

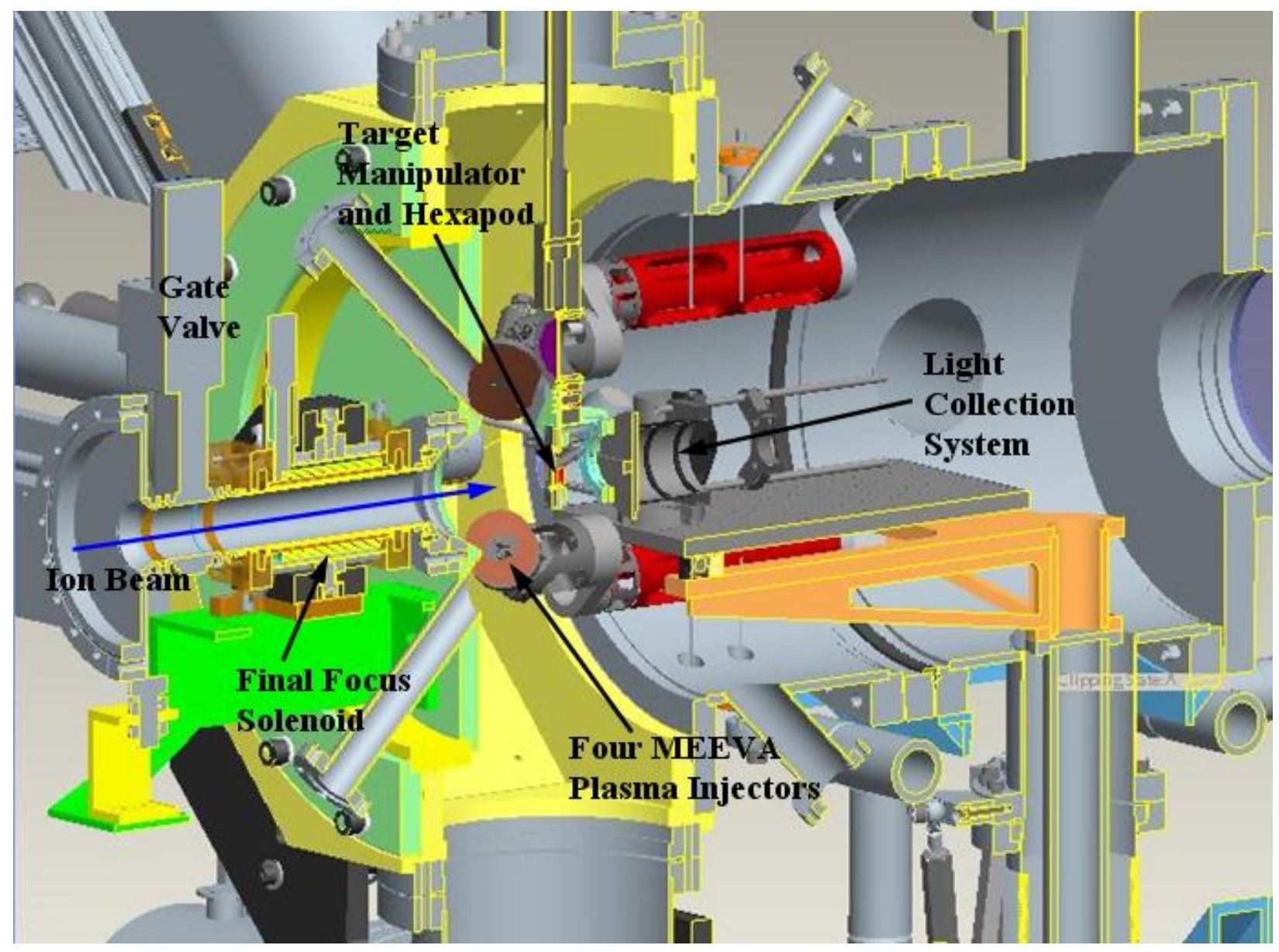

Figure 2: 3D CAD view of the target chamber. The target foil is mounted in the exact center of the chamber with the ports aiming at the target location.

A small vacuum gate valve in front of the final focus solenoid allows decoupling the target chamber vacuum from the accelerator beamline in order to vent the target chamber for target changes, maintenance, or swapping of diagnostics. The bottom chamber ports are reserved for two vacuum pumps, a $1000 \mathrm{l} / \mathrm{sec}$ cryopump with gate valve and a $500 \mathrm{l} / \mathrm{sec}$ turbo pump, an ion gauge and a vent port. 
Design and operation of the 8-Tesla final focus solenoid magnet is described in detail in Ref. 7. The solenoid has operated reliably for plasma injection and beam characterization studies.

Beam charge neutralization in the target chamber is provided by four filtered cathodic arc plasma source (FCAPS) plasma guns used to inject plasma into the final focus region right in front of the target [8]. Significant effort has been expended to minimize the size of the target holder and ion beam diagnostics footprint in order to maximize the plasma flow to the target area. The plasma flow is slowed down once entering the high field region of the final focus solenoid and becomes confined in front of the target.

We are investigating the properties of a gold cone for focusing the ion beam on target. The cone has been modeled with the TRIM code and prototypes tested on the $300-\mathrm{keV}$ potassium NDCX-1 beam. The potential advantages of the cone include its focusing capability due to the inward reflection onto the target of beam particles striking the wall of the cone, enhanced generation of secondary electrons for beam space charge neutralization due to the grazing incidence of beam ions on the cone, reduced space charge due to the presence of the conducting cone wall, homogenizing the energy flux on target, and masking of the portions of the target not under observation by the target diagnostics from unwanted heating by the outer edge of the beam. 


\section{ION BEAM DIAGNOSTICS AND TARGET MANIPULATOR}

Two target chamber ports are reserved for ion beam diagnostics and target manipulation. A fast Faraday cup is mounted on the horizontal vacuum motion-feedthrough. It is a modified design, with better electrical noise isolation against the nearby signals generated by the cathodic-arc plasma sources (the housing is entirely constructed of metal parts) and the design is more modular to facilitate replacing the hole plates with plates of different geometric transparency. The transverse dimensions of both the scintillator and Faraday cup were reduced to fit in the smaller space between the new FCAPS sources (Fig. 2). A scintillator plate and the target holder are mounted on a vertical $x$-y motion stage allowing movement in and out of the target chamber as well as movement along the beam axis.

Since the target foil is less than a micron thick the correct positioning of the target is particularly critical. It must be aligned to both the incoming ion beam and the whole array of target diagnostics. We designed a manipulator with the future development of a target robot system in mind. For a WDM user facility the goal is to have a robotic target handling system which can automatically choose and position from a number of preloaded target assemblies without breaking vacuum.

The target has to be able to be aligned in the $\mathrm{x}-, \mathrm{y}-$, and $\mathrm{z}$-directions, as well as in roll, pitch, and yaw with respect to the ion beam axis. The most compact device for this type of positioning is a "parallel kinematic manipulator", or hexapod. Our current target manipulator consists of such an arm with a manually adjustable hexapod on top. A long vacuum translational feedthrough allows retracting the target from the target chamber center in order to be able to insert ion beam diagnostics. Once the ion beam has been tuned and diagnosed for a particular set of target experiments, the target manipulator moves the target holder into the center of the target chamber.

The miniature hexapod and the beam diagnostics must be compact to minimize shadowing of the injected neutralizing plasma flow. At the same time it has to allow visual access to the side, back, and front of the target foil. No such hexapod is commercially available. We have decided to develop our own system by first implementing a manually adjustable hexapod. We will assess the usefulness our chosen design strategy and improve it for an eventually fully automated system. Currently, in order to replace target foil without requiring a time-consuming cooling and reheating of the ion source, the target chamber can be isolated from the upstream beamline vacuum with a gate valve and vented to air. As a next step we plan to incorporate a vacuum load lock system to facilitate a quicker target replacement time. 


\section{TARGETS}

The target is mounted in the center of the octagonal shaped target chamber and has diagnostic access from the front, rear and side. Initial experiments will provide an opportunity to gain experience with delivering the beam at final focus, as well as with diagnostics on beam-heated WDM targets.

Initial experiments focus on characterizing the NDCX-1 beam at final focus. In these studies the beam will be imaged from the rear on a solid scintillator using a PIMAX camera, or a phototube, or the WDM target diagnostic fiber optic system described below. Initially we plan to use the same alumina scintillators that we have used extensively for beam characterization in our experimental. work. Alumina is known to be relatively robust to beam damage, and previous measurements show sufficiently rapid response to follow the beam intensity on target on the nanosecond time scale. If alumina does not survive the intense beam pulses, or if it does not exhibit a linear response at very high intensity, we have some (5-mm diameter) diamond targets that may prove to be more robust than alumina. Further measurements can be made using the gas cloud from a hole plate, as well as gas-based diagnostics based on beam-induced fluorescence on noble gases such as xenon.

After characterizing the beam, a WDM target will be installed at the focal point of the beam. Targets in hand include foils (350-nm Al, 150-nmAu, 120-nm Pt and 400-nm Si) supported by a wire mesh and thin films deposited on a sapphire substrate (350-nm Al, 150-nm Au and 150-nm $\mathrm{W})$. These targets have a thickness comparable to the range of the $300-\mathrm{keV} \mathrm{K} \mathrm{K}^{+}$beam from NDCX-1, so that the energy deposition distribution is nearly uniform. Simulations using HYDRA of the temperature distribution in typical targets were performed for beam energy delivered to the target by a 2-A, 2-ns, $1-\mathrm{mm}$ diameter, $350-\mathrm{keV} \mathrm{K}^{+}$beam. This corresponds to an energy density of $0.1 \mathrm{~J} / \mathrm{cm}^{2}$. In these calculations tin (Sn) was used in place of gold in order to be able to use the QEOS equation of state tables, and the target reached temperatures around $2400 \mathrm{~K}$. In general the temperature of a target increases as the target atomic mass increases, so that the gold and tungsten targets are expected to reach a higher temperatures.

The length of the NDCX beam pulse can be varied up to at least $10 \mu \mathrm{s}$, and the timing of the compressed portion of the pulse is arbitrary with respect to the beam pulse. The studies mentioned above indicate that foil targets have an important advantage over thin film targets deposited on a substrate. In the case of thin film targets we can take advantage of the beam prepulse before the compressed pulse arrives at the target to slowly heat the target (over 1-10 $\mu \mathrm{s}$ ) through the solid and liquid phase, for example. Since the amount of energy deposition in the pre-pulse may be a factor of 10 or larger than the energy deposition in the compressed pulse, this is an important advantage especially in the initial target experiments. When the target is solid or liquid, minimal expansion or motion of the target is expected on the microsecond time scale.

Figure 3 show time profiles of the temperature for several targets obtained from heat transfer calculations based on a simple model of the heating process where we assume that (neglecting cooling from thermal electron emission, which is expected to be small at this temperature) the target will melt and surface-evaporate until it reaches the flux equilibrium condition where the energy evaporation rate flux $\left(L n v_{T} \exp (-L / k T)\right)$ equals the beam energy flux. Here $L$ is the latent 
heat of vaporization per atom, $\mathrm{kT}$ is the target temperature, $\mathrm{n}$ is the number density of the target, and $\mathrm{v}_{\mathrm{T}}$ is the thermal velocity. The estimated fluence of the uncompressed part of the NDCX pulse is $35 \mathrm{~mJ} / \mathrm{cm}^{2} / \mu \mathrm{s}$.

After heating the target using the beam prepulse the short 1-2 ns compressed pulse can be used more efficiently if the beam pulse is timed such that the target is already at or near the boiling point before the compressed pulse arrives. Rapid heating into the two-phase liquid-gas region is a region of interest in planned target experiments. In the case of deposited film targets, much of the pre-pulse energy is conducted away into the substrate, significantly reducing the effectiveness of this strategy.

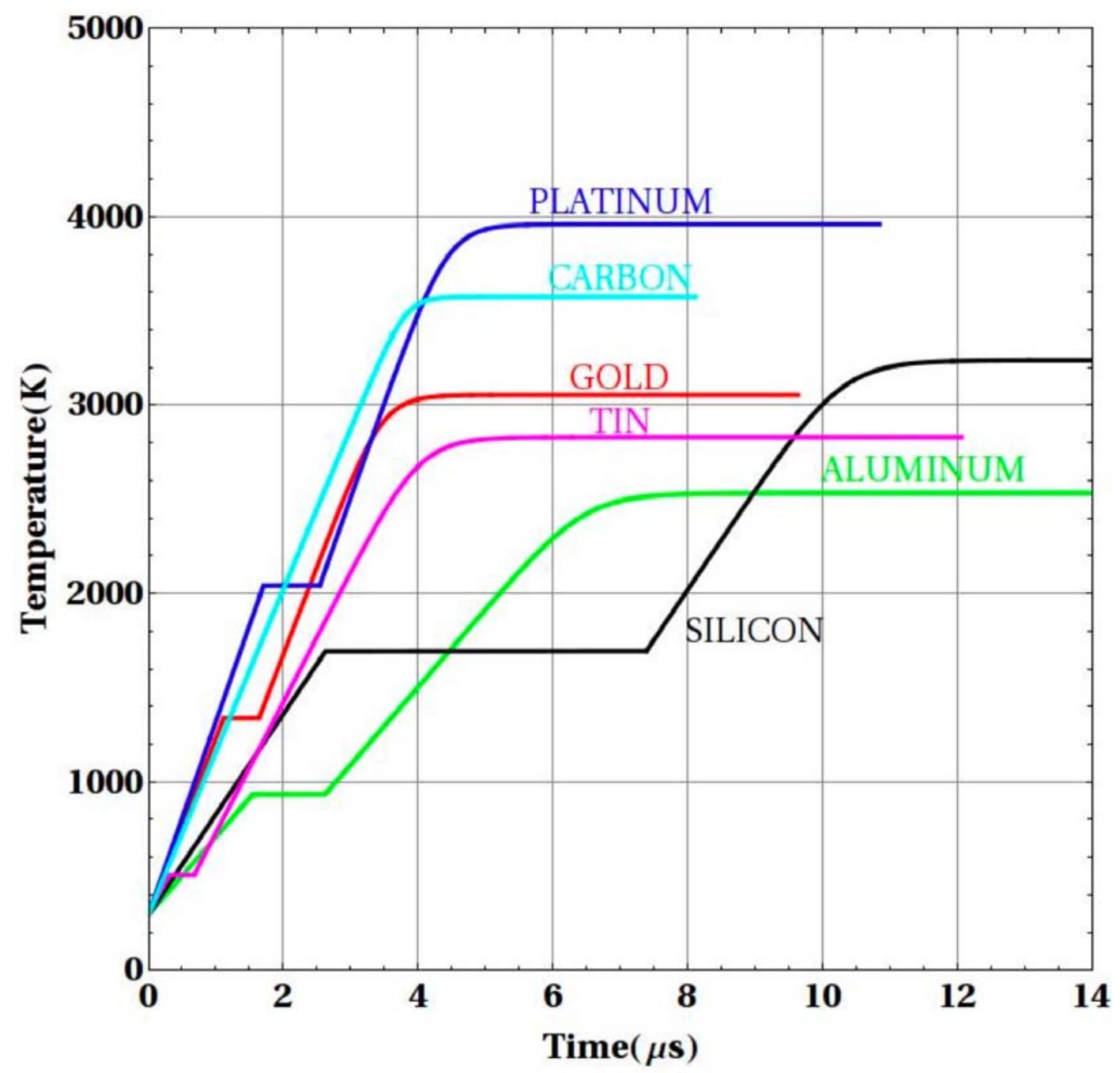

Fig. 3. Time profiles of the temperature for several targets obtained from heat transfer calculations. The sharp transition to constant temperature represents the start of the melting process, and the final temperature is reached when the energy evaporation rate flux equals the beam energy flux. 


\section{TARGET EXPERIMENTS}

As WDM regimes become accessible, experiments will explore the thermodynamic properties of matter heated by the ion beam pulse. These experiments may include solid-liquid and liquidvapor boundary experiments in metals to study two-phase dynamics, the liquid-vapor phase diagram, the equation of state, and droplet formation. The dynamics of the target material as it passes through the phase transition are of interest, and the critical points of many materials remain unknown. The NDCX-1 beam is characterized by a relatively short range in the target material and modest beam intensity on the order of $0.1 \mathrm{~J} / \mathrm{cm} 2$. Future experiments on the NDCX-2 will provide longer beam range and higher beam intensity on target and thus will provide a wider range of possible experiments.

High electron affinity WDM targets

A proposed experiment studies the unique properties of a dense electron-free positive-negative ion plasma $[10,11]$. Halogens $(\mathrm{F}, \mathrm{Cl}, \mathrm{Br})$ are characterized by large electron affinity (3.35-3.6 $\mathrm{eV}$ ). Heating the foil to approximately $0.4 \mathrm{eV}$ with a compressed ion beam pulse produces the halogen WDM. Because of the large electron affinity, a novel state of matter may be obtained, characterized by the presence of a plasma with positive ion balanced predominantly by negative ions with the relative absence of free electrons. This state may exhibit unusual conductivity properties because conduction is ionic. Target parameters and diagnostics of interest include electrical conductivity, beam energy loss, target temperature, etc. Initial operation may utilize gold foils which are expected to be easier to work with than a layer of deposited halogen material. Gold has a relatively large electron affinity $(2.3 \mathrm{eV})$ and the formation of a plasma with positive and negative ions is expected.

\section{Porous targets}

Porous targets have the advantage that the ion beam range is longer than in a solid-density target, thus slowing down the hydrodynamic expansion time of the heated target. We have conducted an experiment at the HHT target station at GSI $[12,13]$. The experiment studied the effect of pore size on target behavior using existing diagnostics, for example by measuring the target temperature as a function of pore size and compare with model predictions of the physics of porous targets. A matter of particular interest is the isotropization of the pore walls as they are heated by the beam, which is expected to scale with the pore size. For example, target dynamics may be affected if the hydrodynamic expansion time to fill in the cells is longer than the beam pulse length.

Other specific near-term experiments of interest include beam induced transient emission and absorption in transparent insulators, conductivity measurements, micro-implosions driven by the ion beam, and thin target $\mathrm{dE} / \mathrm{dx}$ effects on the ion beam. Future experiments on NDCX-2 will explore the WDM regimes at higher temperature than can be achieved in NDCX-1. Other matters of interest under exploration include beam-compression wave coupling in direct-drive cryogenic targets and EOS studies. 


\section{TARGET DIAGNOSTICS}

The primary target diagnostics are a fast pyrometer for temperature measurements, streak camera, spectrometer, VISAR and an optical camera for viewing the target from the rear. A schematic of the setup of the light collection system for these diagnostics is shown in figure 4 .

The collection optics is based on two achromatic lenses (Rayleigh lens system). It has a coupling efficiency $\sim 80 \%$ and allows for direct imaging of the target using a pellicle 1 micron beam splitter and an image guide (to help in alignment and finding the beam spot), as shown in Fig. 4. A drawback of any glass lens system are chromatic aberrations, resulting in wavelength dependence of the probing spot. It has been measured that the probing spot at $1000 \mathrm{~nm}$ is $5 \%$ bigger than at $750 \mathrm{~nm}$. The effect of aberrations with the 400 micron fiber is negligible if the target's temperature is homogenous within a 500 micron. If a smaller fiber (e.g. 100 micron) is used then the optics views the target as an infinite homogenous plane and aberration are not a concern.

$2 \times$ NIR achromatic doublets, $f=75, d=50.8$

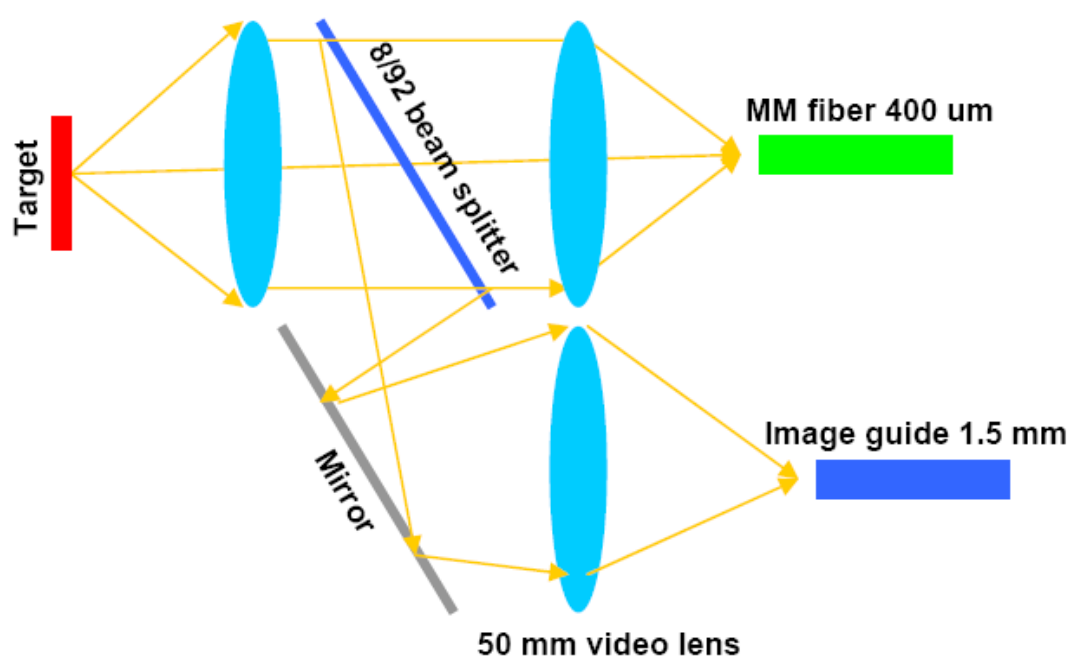

Figure 4. Schematic of optics of light collection system. The 400 micron fiber carries the optical signal to the pyrometer; the image guide provides an image of the beam spot on target to a camera.

\subsection{Pyrometer}

A fast optical pyrometer [7] has been developed and commissioned on a laser-based WDM experiment at the ALS. It is ready for the upcoming beam experiments. The main challenge when compared to the pyrometer of Ref. 7 is the extremely fast (sub-ns) time resolution and higher sensitivity, i.e. ability to measure T from $1500 \mathrm{~K}$ to $5000 \mathrm{~K}$ (at GSI these values are $5 \mathrm{~ns}$ resolution $\mathrm{T}$ from $2000 \mathrm{~K}$ to $20000 \mathrm{~K}$ ). In order to fulfill these requirement, much higher 
efficiency spectral resolution, optical beam transport and appropriate type of detectors were investigated.

The working scheme is shown in Fig. 5. We use custom broad-band-spectrally-selective beam splitters which have transmission at the level of $\sim 90 \%$ (interference filters have $30 \%-70 \%$ ) in order to maintain high sensitivity of the system.

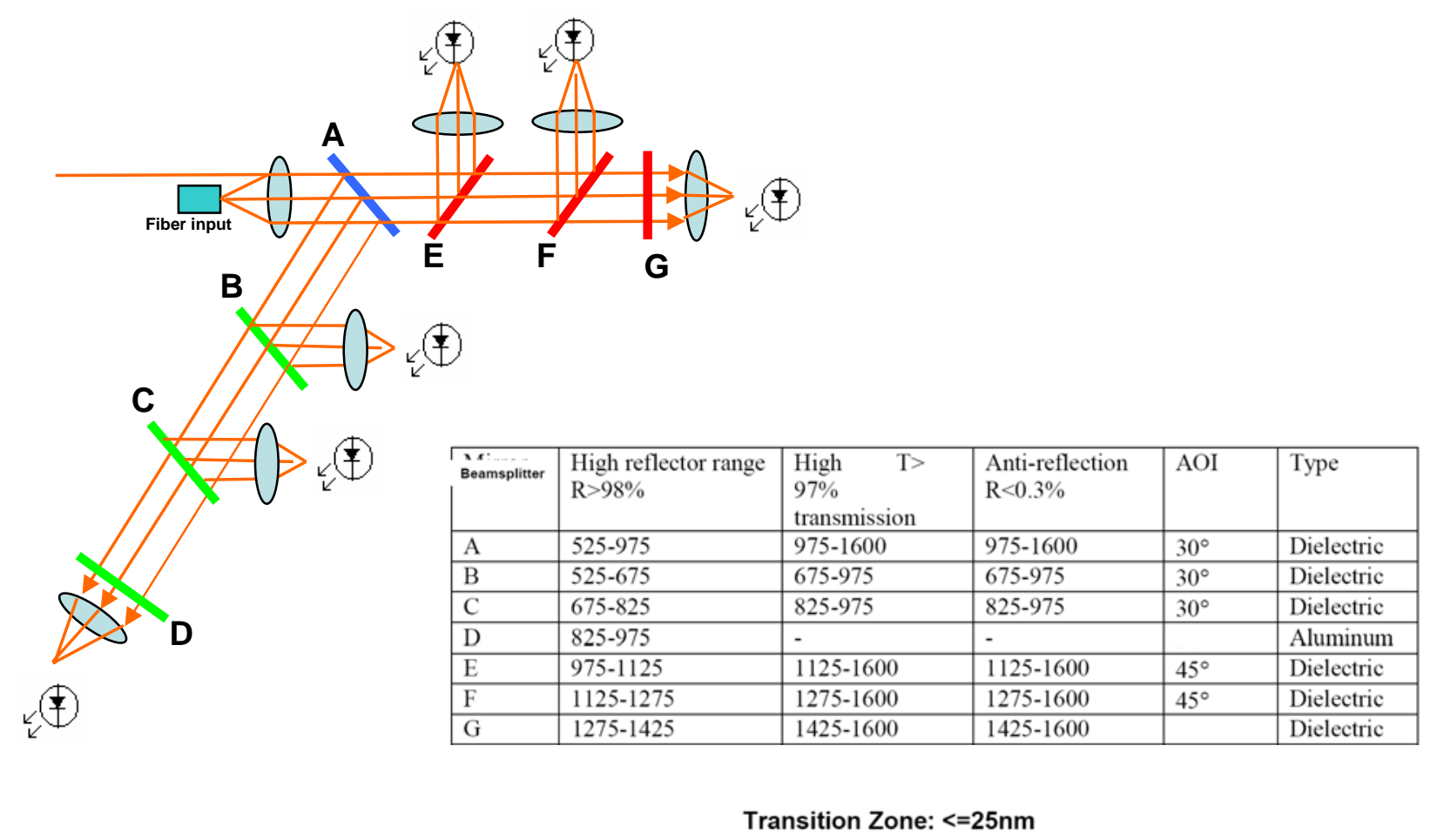

Figure 5. Optical working scheme of pyrometer. The specifications of the beam splitter/filters are indicated in the table. 
A photo-detector sensitive to $\sim 1 \mathrm{~mW}$ of light from $400 \mathrm{~nm}$ to $2000 \mathrm{~nm}$ in a sub-ns time range is required. In addition, an extremely broad band and flat amplification gain curve from DC to 4 $\mathrm{GHz}$ is necessary in order to be able to calibrate the pyrometer with DC tungsten filament lamp and at the same time detect temperature behavior features on a sub-nanosecond time scale. Also a relatively big $(0.5 \mathrm{~mm})$ detector size for a simplified alignment is desirable.

We have tested several options, including PIN photo receivers and photomultiplier tubes (PMT). A photo receiver was chosen due to its flat gain, $75 \mathrm{ps}$ response time, and a reasonable price, however these detectors are fiber coupled which is associated with losses in efficiency. Two channels are indicated in Fig. 5. Recently we have installed the third channel, and more channels will be added in the future.

Calibration of the pyrometer is carried out with a tungsten ribbon lamp ( $\mathrm{T}=2600 \mathrm{~K})$, and produces $2 \mathrm{mV}$ voltage at $\mathrm{C}$ channels and $5 \mathrm{mV}$ at $\mathrm{E}$ channel. Noise rms is $0.05 \mathrm{mV}$. Saturation voltage (non-linear regime) is $1.8 \mathrm{~V}$.

The pyrometer has been tested in a laser-driven WDM experiment at one of the beamlines of the Advanced Light Source (ALS) at LBNL [8]. These results indicate that the pyrometer as fielded at ALS has resolution down to $\leq 2000 \mathrm{~K}$. 


\subsection{Streak camera/spectrometer}

Our C7700 Hamamatsu streak camera was bench tested using a pulsed LED. In addition, we used the camera in conjunction with a Horiba Jobin Yvon spectrometer grating (PAC CP140104 CP140 - 104 Spectral range : 250 - $850 \mathrm{~nm}$ over $24.7 \mathrm{~mm}, 285$ grooves/ mm) for time resolved spectroscopy of the neutralization plasma on NDCX. Fig. 6 shows sample experimental results from this measurement.

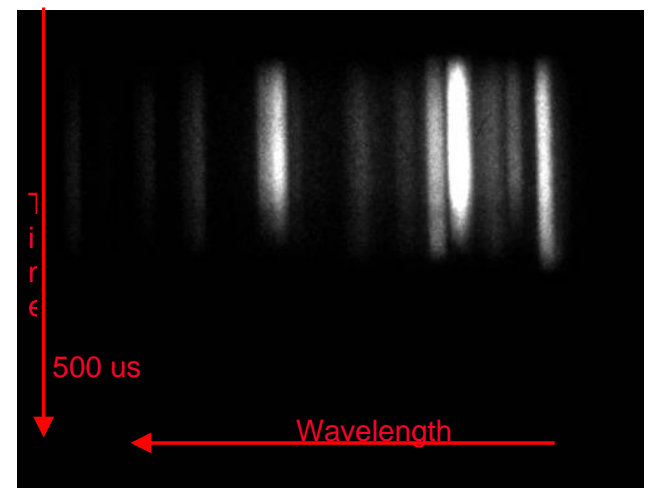

Figure 6. Spectral emission as a function of time and wavelength during a pulse of the aluminum FCAPS plasma in the NDCX target chamber as measured by the streak spectrometer.

\subsection{VISAR}

We have tested our fiber-coupled VISAR (velocity interferometer system for any reflector, Martin Froescher and Associates) using a conventional roofing nail gun. A polished piece of aluminum was glued to the piston tip and the probing laser $(1500 \mathrm{~nm}, 15 \mathrm{~mW})$ spot was focused at it using a supplied $50 \mathrm{~mm}$ lens. We used $11 \mathrm{~m}$ meter delay leg, which provides $50 \mathrm{~ns}$ temporal resolution $0.02 \mathrm{~m} / \mathrm{s}$ velocity resolution. In WDM experiments, we plan to use a $200 \mathrm{~mm}$ delay leg, which will measure velocity with $1 \mathrm{~ns}$ temporal resolution and $4 \mathrm{~m} / \mathrm{s}$ velocity resolution. Also a MATLAB code was written for velocity extraction from the four curves produced by the instrument. We now know how to align, tune the quadrate, operate and process the data of the VISAR. It is unlikely that the VISAR will be used in the first round of WDM experiment, due to low temperature, i.e. the expansion velocity is expected to be relatively small. 


\section{INSTALLATION ON NDCX}

Fig. 7 shows the installation of the target chamber on NDCX-1. The target chamber facility is mounted on linear rails to be able to move it in and out of the beamline for servicing and experimental re-arrangements. In support of the target chamber facility we have installed a separate laser room facility in the floor below the target chamber. The laser room can be used for optical target diagnostics pre-alignment and tests. The whole setup including the laser room will be a core capability for any future WDM user facility in the HIFS-VNL.

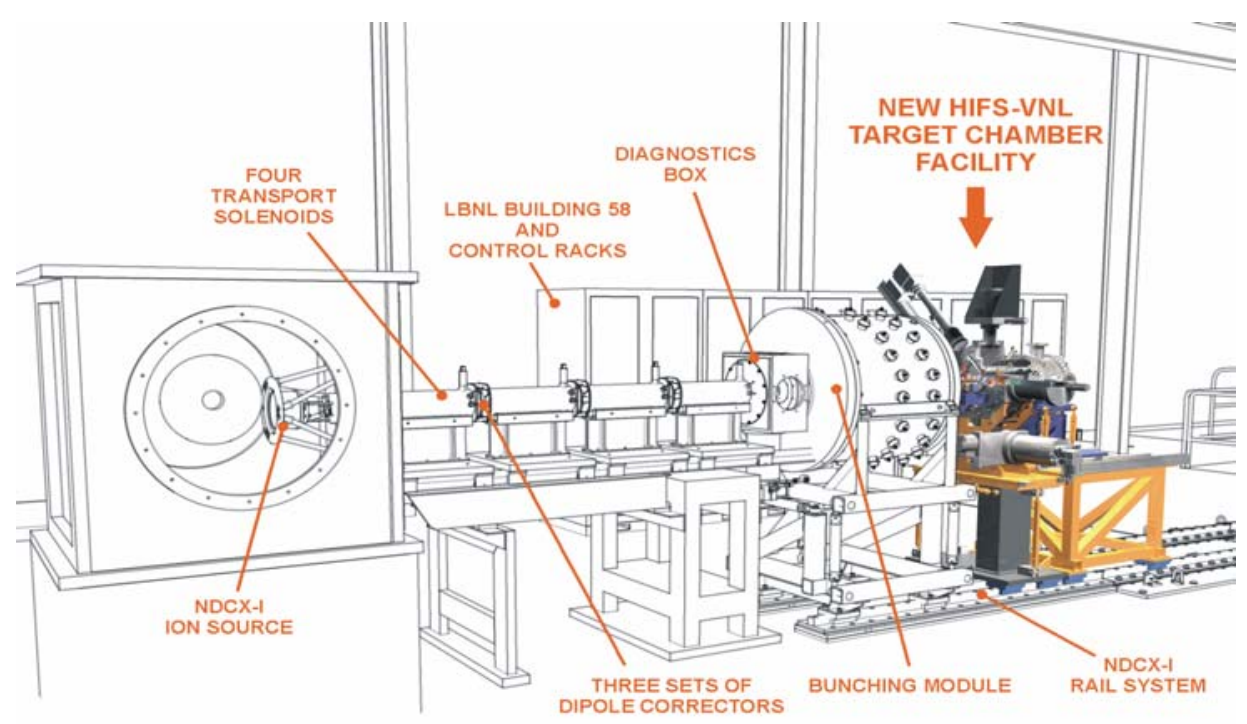

Figure 7. HIFS-VNL target chamber installed on NDCX-1.

\section{SUMMARY}

We have described the target chamber facility, diagnostics and targets now available for target experiments on NDCX-I. The same target chamber facility can also be used on future experiments, e.g. NDCX-II, which could provide significantly more beam power. As future plans for target experiments will evolve we will improve and continually update the target chamber facility to advance the challenging experiments with ion-beam driven, isochorically heated warm dense matter.

* Work performed under the auspices of the U.S. Dept. of Energy by LBNL and LLNL under Contracts No. W-7405-Eng-48 and DE-AC02-05CH11231.

\#fmbieniosek@lbl.gov 


\section{REFERENCES}

1. R.C. Davidson, Chair, Frontiers in High Energy Density Physics, National Research Council of the National Academies, National Academies Press, Washington, DC, USA 2003.

2. B. G. Logan, et.al., Nucl. Instrum. Meth. A 577 (2007) 1-7.

3. L. R. Grisham, Phys. Plasmas 11 (2004) 5727.

4. P.A. Seidl, et al., Plans for longitudinal and transverse neutralized beam compression experiments, and initial results from solenoid transport experiments, NIM A 577 (2007) 215-222.

5. D.H.H. Hoffmann, et al., Present and future perspectives for high energy density physics with intense heavy ion and laser beams, Laser and Particle Beams 23 (2005) 47-53.

6. F.M. Bieniosek, J.J. Barnard, M.A. Leitner, R.M. More, P.K. Roy, Diagnostics for nearterm warm dense matter experiments, NIM A 577 (2007) 284-288.

7. P. Ni, et al., Pyrometric system for temperature measurements of HED matter generated by intense heavy ion beams, Journal de Physique IV 133 (2006) 977-980.

8. P. A. Ni, et al., Overview of planned diagnostics for warm dense matter experiments with intense heavy ion beams, these proceedings.

9. P. K. Roy, et al., A space-charge neutralizing plasma for beam drift compression, these proceedings.

10. R. More, H. Yoneda, and H. Morikami, JQSRT 99 (2006) 409-424.

11. L.R. Grisham and J.W. Kwan, Perspective on the role of negative ions and ion-ion plasmas in heavy ion fusion science, these proceedings.

12. D. Varentsov, et al., High energy density physics experiments with intense heavy ion beams, Nucl. Instrum. Meth. A 577 (2007) 262-266.

13. B. G. Logan, et al., Heavy ion fusion science research for high energy density physics and fusion applications, IFSA07, Kobe, Japan, September 9-14, 2007. 